\title{
Putting down the pineal gland in zebrafish
}

Circadian rhythms are a critical part of normal biology, as evidenced by virtually all animals displaying a roughly 24 -hr cycle of key physiological functions. Although typically studied at higher behavioral levels in vertebrates, like sleep-wake cycles, recent research indicates that circadian rhythms can have important effects at the cellular and network level, and can influence a wide-range of systems processes, including immune function and cancer development.

Owing to these facts, scientists have consistently tried to crack the mechanisms driving circadian rhythms in whole organisms. In mammals, the master circadian clock is located in the suprachiasmatic nucleus ( $\mathrm{SCN}$ ), with individual SCN neurons independently capable of maintaining rhythmic changes in activity owing to a precise molecular network of genes switched on and off over a roughly $24-\mathrm{hr}$ cyclic period. However, despite a relatively clear picture of the molecular framework underlying cellular circadian

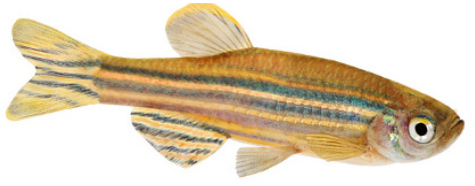

zebrafish, the master circadian clock has traditionally been thought to reside in the pineal gland, but recent results demonstrating clock function in other tissue has brought this view into question. To further assess the role pineal rhythms, a systems level understanding is only beginning to come into focus. For instance, although the SCN is necessary to maintain circadian rhythms, it is also known that multiple tissues can maintain their own independent clocks, suggesting the SCN may act more akin to a conductor guiding a larger orchestra of rhythms. Understanding how circadian rhythms arise and are maintained at this systems level can be difficult in mammalian models, and some researchers turn to other model organisms.

One group of researchers, led by Yoav Gothilf at Tel-Aviv University, has recently applied a genetic approach in zebrafish to generate a novel knock-down model to better understand circadian function in this species (PLoS Genetics 12, e1006445; 2016). In cells play in driving circadian rhythms, the group developed a novel model where melatonin expressing pineal cells had their molecular clock selectively blocked with a dominantnegative strategy. The group found evidence that circadian function in locomotor and place preference had been disrupted, but other tissues outside the pineal gland maintained their core clock function. Overall the results suggest that the pineal gland plays an important role in modulating rhythmic behaviors, but may be part of a multi-pacemaker distributed system governing organismal-level circadian function. Importantly, the novel transgenic model developed in this work may help future studies uncover important mechanisms for multi-system clock function.

Dustin M. Graham 